\title{
Prevalence and associated risk factors of human intestinal parasitic infections: a population-based study in the southeast of Kerman province, southeastern Iran
}

Mohammad Javad Abbaszadeh Afshar ${ }^{1 *}$, Maryam Barkhori Mehni ${ }^{2 *}$, Mostafa Rezaeian ${ }^{1}$, Mehdi Mohebali ${ }^{1}$, Vali Baigi ${ }^{3}$, Somayeh Amiri ${ }^{2}$, Mohammad Bagher Amirshekari ${ }^{2}$, Ruhollah Hamidinia ${ }^{2}$ and Mohammad Samimi ${ }^{2}$

\begin{abstract}
Background: Determination of the prevalence and distribution pattern of intestinal parasites is a fundamental step to set up an effective control program to improve the health status. This study aimed to determine the prevalence of intestinal parasitic infections and associated risk factors among inhabitants of Rudbar-e Jonub county, southeast of Kerman province, southeastern Iran.

Methods: In this cross-sectional study, 861 stool specimens were collected from inhabitants of Rudbar-e Jonub county through a multistage cluster sampling method in 2018. The collected specimens were examined by parasitological methods including, direct wet-mounting (for the fresh specimens with a watery consistency), formalin-ethyl acetate sedimentation and agar plate culture.

Results: The prevalence of intestinal parasites was 34.2\% (95\% Cl 30.1 to 38.2). The prevalence of protozoan parasites 32.3\% (95\% Cl 28.4 to 36.5) was significantly higher than helminthic parasites 3.2\% (95\% Cl 2.1 to 4.7 ). Blastocystis sp. (13.3\%), Entamoeba coli (11.4\%) and Giardia lamblia (10.6\%) as protozoan parasite and Hymenolepis nana (2.4\%) as helminthic parasite were the most common detected intestinal parasites in the study. Entamoeba histolytica/dispar (1.5\%), lodamoeba bütschlii (1.0\%), Chilomastix mesnili (0.5\%), Entamoeba hartmanni (0.4\%), Enterobius vermicularis (0.3\%) and Ascaris lambercoides (0.3\%) were other detected parasites. Multiple logistic regression revealed a significant association of intestinal parasitic infections with source of drinking water and residency status (rural/urban). Multiple infections with 2 or 3 parasitic agents constituted 22.7\% of 295 infected cases.
\end{abstract}

Conclusions: This study revealed a high prevalence of intestinal protozoan infections among inhabitants of Rudbar-e Jonub county. Intestinal parasites especially protozoans remain a challenging public health problem wherever sanitation and health measures are limited in Iran.

Keywords: Intestinal parasites, Human, Prevalence, Iran

\footnotetext{
* Correspondence: Mj.afshar1990@gmail.com; M.bmehni1980@gmail.com

${ }^{1}$ Department of Medical Parasitology and Mycology, School of Public Health,

Tehran University of Medical Sciences, Tehran, Iran

${ }^{2}$ Health Affairs, Jiroft University of Medical Sciences, Jiroft, Iran

Full list of author information is available at the end of the article
}

(c) The Author(s). 2020 Open Access This article is distributed under the terms of the Creative Commons Attribution 4.0 International License (http://creativecommons.org/licenses/by/4.0/), which permits unrestricted use, distribution, and reproduction in any medium, provided you give appropriate credit to the original author(s) and the source, provide a link to the Creative Commons license, and indicate if changes were made. The Creative Commons Public Domain Dedication waiver (http://creativecommons.org/publicdomain/zero/1.0/) applies to the data made available in this article, unless otherwise stated. 


\section{Background}

Despite the advancement in sanitation infrastructure and hygiene status, intestinal parasitic infection remains a considerable public health problem, especially in developing countries [1]. It is estimated that more than three billion people (mostly children) are infected with intestinal parasites around the world [1, 2]. In Iran, due to diversity in socioeconomic, geographic, sanitary/hygiene, cultural, and educational status a broad range of intestinal parasites prevalence between 4.7 to $56 \%$ have been reported in the apparently healthy populations $[3,4]$. Because of low socioeconomic status, limited sanitation, and also geographic factors, rural areas are regarded as endemic areas of intestinal parasitic infections in the southern part of Iran $[5,6]$.

Determination of the prevalence and distribution pattern of intestinal parasitic infections is a fundamental step to set up a prevention and control program to improve the health status. On the other hand, due to diversity in geographic factors and socio-cultural patterns in different parts of Iran, an epidemiological study is required in each region separately. To our knowledge, there is no study available on the distribution of intestinal parasitic infections in Rudbar-e Jonub county as a tropical area and with a deprived community in southeast of Kerman province, southeastern Iran. Therefore, this study conducted to determine the prevalence and risk factors associated with intestinal parasitic infections in Rudbar-e Jonub county inhabitants.

\section{Methods}

\section{Study area}

Rudbar-e Jonub county with an area about $7000 \mathrm{~km}^{2}$ located in "Hamun-e Jaz Murian" wetland basin, southeast edge of Kerman province, southeastern Iran $\left(28^{\circ} 01^{\prime} 45.5^{\prime \prime}\right.$ $\mathrm{N} 57^{\circ} 59^{\prime} 34.8^{\prime \prime} \mathrm{E}$ ). It is comprised of two districts and four rural districts. Based on information of the Statistical Center of Iran represented in 2016, Rudbar-e Jonub has a population of 105,992 inhabitants in 27,428 households. About $80 \%$ of the population settled in rural areas. This area has a warm and semi-arid climate (Fig. 1) [7].

\section{Study design}

This cross-sectional study was conducted in Rudbar-e Jonub county in 2018. Eight hundred and sixty-one (861) stool specimens were collected through a multistage cluster sampling from 4 rural districts of the county as the study strata. In each rural district, health centers selected through proportional-to-size random sampling. A total of 30 health centers were selected throughout the county. Ten households covered by each health center were selected using the systematic sampling approach. All members of the selected households were invited to take part in the study. If individuals in a household refused to take part, the next household was invited. A pre-designed checklist including sex, age group, occupation, education level, source of drinking water, type of residency, and animal close contact was filled for each participant. Out of a total of 1500 individuals approached, $42.6 \%$ refused to give the sample (Response rate $=57.4 \%$ ).

\section{Sample collection and laboratory analysis}

Stool specimens were collected in the pre-labeled, widemouth, plastic containers. At the laboratory section of the health centers after examining the specimens for consistency, color, the presence of blood, mucus and

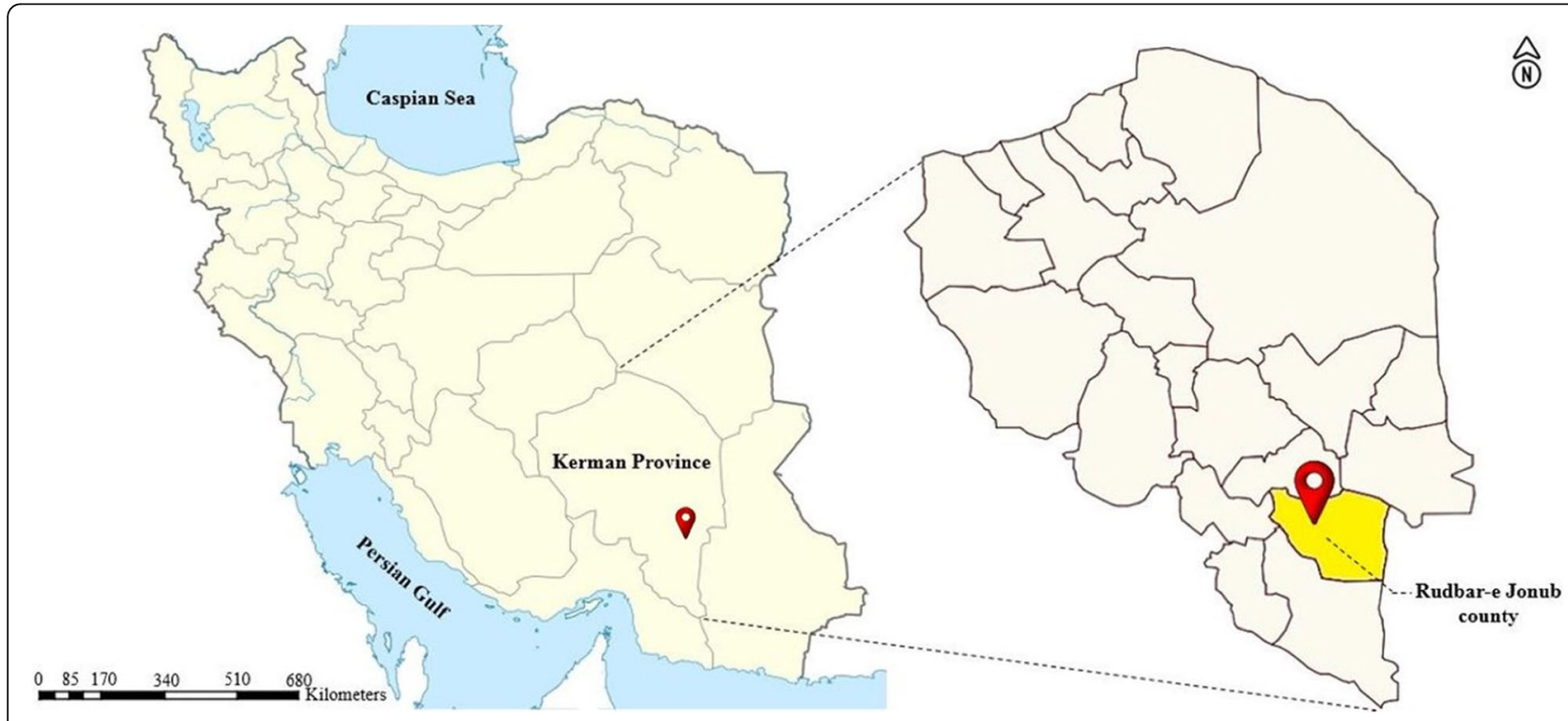

Fig. 1 Map of the study area. Left: Map of Iran, Right: Location of Rudbar-e Jonub county in Kerman province 
adult intestinal helminths, macroscopically, a direct wetmount was prepared and examined for the fresh specimens with a watery consistency or containing blood or mucus under low-power objective $(10 x)$ and high dry objective $(40 \times)$ for suspicious objects. Also, a part of each collected specimen (approximately $2 \mathrm{~g}$ ) was cultured on agar plates. Then, all specimens were preserved in $10 \%$ buffered formalin and transported to the laboratory affiliated to Jiroft University of Medical Sciences for formalin-ethyl acetate sedimentation method. Merthiolate-iodine-formaldehyde (MIF) solution was used to temporary staining of sediments obtained from the formalin-ethyl acetate method. The specimen collection, processing, shipping, and the parasitological methods were carried out as described by Garcia et al. [8]. All microscopic evaluations and identification were made by the same observer(s) blinded to participants information. Specimens were considered positive if the helminth eggs, larvae, or cysts and/or trophozoites of protozoans were detected by at least one of the three methods.

\section{Statistical analysis}

The frequency was calculated for qualitative and categorical variables. The crude and adjusted associations between intestinal parasitic infection and determinants were assessed using univariate and multiple logistic regressions. To adjust for the population distribution, poststratification corrections were made to sampling weights. Statistical significance was accepted at $p$ values $<0.05$. Statistical analysis was done using Stata v.14.2 (Stata Corp LP, Texas, USA).

\section{Results}

Over 1 year, 861 stool specimens from the Rudbar-e Jonub participants including 400 (46.1\%) males and 461 (53.9\%) females were collected. The majority of the participants were children up to 9 years of age (25.9\%). Only $6.9 \%$ of the participants had an academic education and $20.5 \%$ of them were illiterate. $35.6 \%$ of the participants had no safe drinking water (Table 1).

At least one species of the intestinal parasites was found in $34.2 \%$ (95\% CI 30.1 to 38.2) of the participants. Multiple infections with 2 or 3 parasitic agents constituted $22.7 \%$ of 295 infected cases. Any parasitic agent was seen in direct examination (performed on the samples with a watery consistency) and also agar plate culture method. The prevalence of detected intestinal parasites by formalin-ether sedimentation method embedded in Table 2. The prevalence of protozoan parasites $32.3 \%$ (95\% CI 28.4 to 36.5 ) was significantly higher than helminthic parasites $3.2 \%$ (95\% CI 2.1 to 4.7). Blastocystis hominis, Entamoeba coli and Giardia lamblia were the most common intestinal protozoan with a prevalence of 13.3 (95\% CI 11.0 to 15.5 ), 11.4 (95\% CI 8.7 to 15.4 ) and 10.6 (95\% CI 8.5 to 13.1 ), respectively. Entamoeba histolytica/dispar, Iodamoeba bütschlii, Chilomastix mesnili and Entamoeba hartmanni were other detected protozoan parasites in the study. Also, the most prevalent helminthic infection was Hymenolepis nana with a prevalence of 2.4 (95\% CI 1.5-3.9). Ascaris lumbricoides and Enterobius vermicularis were other detected helminthic parasites.

The results of unadjusted and adjusted logistic regression analyses of the risk factors associated with intestinal parasitic infections among the participants embedded in Table 3. Among possible risk factors investigated in this study, the source of drinking water and residency status (rural/urban) were found to have a significant association with intestinal parasitic infections $(p$ value $<0.05$ ) There was no association between the infection and sex, age group, occupation, education level, and animal close contact.

\section{Discussion}

The results of this study showed one-third (34.2\%) of the inhabitants in Rudbar-e Jonub were infected by intestinal parasites. This finding is consistent with the studies carried out in apparently healthy inhabitants in recent decades in rural and tribal areas of the country. Barkhori et al. [9] reported $28 \%$ of the infection in Jiroft district, near the studied area. Similarly, a relatively high prevalence of the infection in nomadic tribes of Khuzestan province $(25.4 \%)$ and rural inhabitants of Mazandaran (25\%), Kohgiluyeh and Boyer-Ahmad (37.5\%), Lorestan (32.5) and Hamadan (35.1\%) provinces has been reported [10-14]. Also, Hemmati et al. [15] in a study on inhabitants of Rudehen in Tehran province, capital of Iran, have reported $32.7 \%$ of the infection. Besides, some studies reported a significant prevalence of $48.8 \%$ in rural inhabitants of Hormozgan [6] and 56\% in nomadic tribes of Chaharmahal and Bakhtiari provinces [4].

The finding of the current and aforementioned studies reflects the fact that in spite of advances in sanitation and personal/public health measures, it seems there is still not enough arrangements for controlling of intestinal parasites and these neglected agents especially protozoans are still a significant public health problem in rural and tribal areas of the country. The importance of this issue will become clear when we know these reported prevalences seem to be less than the actual value because in most studies on intestinal parasites prevalence only one stool specimen of subjects was collected for examination whereas for a standard diagnosis collecting three sequential specimens in three alternate days is required [16]. Also, in most studies, no specific methods such as modified acid-fast staining and Graham test for diagnosis of coccidia and $E$. vermicularis respectively, are performed. 
Table 1 Socio-demographic characteristics of participants $(n=861)$

\begin{tabular}{|c|c|c|c|}
\hline Characteristics & Number & Percent (un-weighted) & Percent (weighted) \\
\hline \multicolumn{4}{|l|}{ Sex } \\
\hline Male & 400 & 46.5 & 46.1 \\
\hline Female & 461 & 53.5 & 53.9 \\
\hline \multicolumn{4}{|l|}{ Age group } \\
\hline$\leq 9$ & 234 & 27.2 & 25.9 \\
\hline 10 to 19 & 151 & 17.5 & 16.7 \\
\hline 20 to 29 & 134 & 15.6 & 15.5 \\
\hline 30 to 39 & 156 & 18.1 & 18.4 \\
\hline 40 to 49 & 98 & 11.4 & 11.5 \\
\hline $50 \leq$ & 88 & 10.2 & 12 \\
\hline \multicolumn{4}{|l|}{ Occupation } \\
\hline Housewife & 222 & 25.8 & 26.6 \\
\hline Children & 132 & 15.3 & 13.2 \\
\hline Student & 202 & 23.5 & 24.2 \\
\hline Farmer & 136 & 15.8 & 15.7 \\
\hline Employed & 70 & 8.1 & 8.9 \\
\hline Unemployed & 24 & 2.8 & 2.5 \\
\hline Others & 75 & 8.7 & 8.9 \\
\hline \multicolumn{4}{|l|}{ Education level } \\
\hline Children under 6-yr & 135 & 15.7 & 13.6 \\
\hline Elementary school & 242 & 28.1 & 28.9 \\
\hline High school & 256 & 29.7 & 30.1 \\
\hline University & 55 & 6.4 & 6.9 \\
\hline Illiterate & 173 & 20.1 & 20.5 \\
\hline \multicolumn{4}{|l|}{ Source of drinking water } \\
\hline Tap water & 558 & 64.8 & 64.4 \\
\hline Spring or well water & 303 & 35.2 & 35.6 \\
\hline \multicolumn{4}{|l|}{ Animal close contact } \\
\hline Yes & 501 & 58.2 & 60.1 \\
\hline No & 360 & 41.8 & 39.9 \\
\hline \multicolumn{4}{|l|}{ Residency status } \\
\hline Rural & 751 & 87.2 & 87.7 \\
\hline Urban & 110 & 12.8 & 12.3 \\
\hline
\end{tabular}

Blastocystis sp., E. coli, and G. lamblia were the most common intestinal parasites among the study population similar to other studies in Iran $[6,9-11]$. All of them have a fecal-oral transmission mode, indicates poor hygiene in Rudbar-e Jonub county. According to the current findings, the prevalence of Blastocystis $s p$. infection was found at $13.3 \%$ (95\% CI 11.0 to 15.5). The reported range of Blastocystis infection in the apparently healthy populations in the country varies from 7.5 to $28.4 \%[10,15]$. Several studies have revealed an association between carrying the parasite and some clinical manifestations that is controversial yet [17-19]. In this study, the highest prevalence of Blastocystis infections was observed in the age group of 30 to 39 year. Some studies have suggested that the incidence of Blastocystis infection increases with age $[15,20]$. Due to the unclear aspects of zoonotic, mode of transmission, and potential host factors important for colonization, it seems, discussion about such association needs more evidence.

The prevalence of $E$. coli in the current study was found $11.4 \%$ (95\% CI 8.7 to 15.4). In the studies carried out in recent decade on apparently healthy people in Iran, the prevalence of $E$. coli reported up to $18.9 \%$ [13]. Although E. coli and other non-pathogenic parasites 
Table 2 Prevalence of intestinal parasites in the participants $(n=861)$

\begin{tabular}{|c|c|c|}
\hline Parasite & Number of infected & Prevalence $\left(95 \% \mathrm{Cl}^{\mathrm{a}}\right)$ \\
\hline \multicolumn{3}{|l|}{ Protozoa } \\
\hline Blastocystis hominis & 114 & 13.3 (11.0 to 15.5$)$ \\
\hline Entamoeba coli & 98 & $11.4(8.7$ to 15.4$)$ \\
\hline Giardia lamblia & 92 & $10.6(8.5$ to 13.1$)$ \\
\hline Entamoeba histolytica/dispar & 14 & 1.5 (0.8 to 2.6$)$ \\
\hline lodamoeba bütschlii & 11 & $1.0(0.5$ to 1.9$)$ \\
\hline Chilomastix mesnili & 5 & 0.5 (0.1 to 1.2$)$ \\
\hline Entamoeba hartmanni & 4 & $0.4(0.1$ to 1.0$)$ \\
\hline Total $^{b}$ & 280 & $32.3(28.4$ to 36.5$)$ \\
\hline \multicolumn{3}{|l|}{ Helminths } \\
\hline Hymenolepis nana & 22 & 2.4 (1.5 to 3.9$)$ \\
\hline Enterobius vermicularis & 3 & 0.3 (0.0 to 0.9 ) \\
\hline Ascaris lumbricoides & 3 & $0.3(0.1$ to 1.3$)$ \\
\hline Total & 28 & $3.2(2.1$ to 4.7$)$ \\
\hline Total $^{\mathrm{b}}$ & 295 & 34.2 (30.1 to 38.2$)$ \\
\hline
\end{tabular}

${ }^{\mathrm{a}} \mathrm{Cl}$ confidence interval

${ }^{\mathrm{b}}$ There were also some cases of coinfection with two or three species

detected in this study do not cause infection, their presence indicates the fecal-oral transmission in the host, which is an indicator for the general assessment of the hygiene status of the area. In this study, the prevalence of Giardia infection was 10.6\% (95\% CI 8.5-13.1).

The prevalence of G. lamblia in the apparently healthy populations in Iran has been reported 33.9 and $10.2 \%$ in 2008 [10, 11], 28.2 and $8.9 \%$ in 2009 [4, 14], $17.2 \%$ in 2011 [6], $2.2 \%$ in 2014 [13], 17.4 and $7.8 \%$ in 2016 [9, 12], and $1.2 \%$ in 2017 [15]. The overall prevalence of Giardia has shown a declining trend during the past decade but it appears to be still relatively high depending on the target population.

The prevalence of human helminthic diseases declined sharply in recent decades throughout Iran but some of them, particularly those with direct fecal-oral transmissions, such as Hymenolepis and Enterobius, remain common in some parts of the country [21]. In the current study, the helminthic infection was limited to only three species among which most infection was related to $H$. nana $2.4 \%$ (95\% CI 1.5 to 3.9). Although the prevalence of $H$. nana in human has fallen since 1970 [21] it remains relatively common in the rural an tribal areas of Iran $[4,9,10]$. E. vermicularis with a $0.3 \%$ (95\% CI 0.0 to 0.9) prevalence was another detected helminthic infection. Given that the Graham test was not done in this study, the actual prevalence is probably higher than the reported value. Reduction in the prevalence of these parasites will need more direct interventions, such as the employing experienced technician in medical laboratories and treatment of infected cases and also, health education to informing people from transmission route of these helminthic infections. Also, $0.3 \%$ (95\% CI 0.1 to 1.3) of infection with $A$. lumbricoides as a soiltransmitted helminth (STH) was detected in this study. Due to effective measures to improve public health in Iran the prevalence of $A$. lumbricoides dropped from $46.7 \%$ in 1987 [22], $17.8 \%$ in 1992 [23], and $16.3 \%$ in 1996 [24] to $0.4 \%$ in this study. Low prevalence of intestinal helminthic infections in Rudbar-e Jonub is in concordance with the result of recent studies in other parts of Iran $[9,13,15]$.

In this study, several possible determinants associated with intestinal parasitic infections were investigated and a significant association was found between intestinal parasitic infections and, source of drinking water and residency status (rural/urban). The source of drinking water is an important risk factor for infection with intestinal protozoa such that a waterborne transmission of all detected protozoa in this study is possible. In this study, $35.2 \%$ of the participants were deprived of safe drinking water. Prevalence of intestinal parasites in participants who used non-sanitary drinking water was $38.4 \%$ (95\% CI 32.6 to $44.6 \%$ ), significantly higher than other participants, suggesting the possibility of waterborne transmission. Similar results have been reported in some studies regarding the importance of sources of drinking water $[9,15]$. The need for improvement of public health infrastructure in Rudbar-e Jonub county is evident. About $80 \%$ of the population in Rudbar-e Jonub have a rural lifestyle. In this study, the prevalence of intestinal parasites was higher in rural areas than in urban areas, significantly. Rural lifestyle is itself risky due to insufficient 
Table 3 Univariate and multiple analysis of intestinal parasitic infections and potential risk factors $(n=861)$

\begin{tabular}{|c|c|c|c|}
\hline Risk factors & Prevalence $(95 \% \mathrm{Cl})$ & $\mathrm{OR}^{\mathrm{a}}(95 \% \mathrm{Cl})$ & $\mathrm{OR}^{\mathrm{b}}(95 \% \mathrm{Cl})$ \\
\hline \multicolumn{4}{|l|}{ Sex } \\
\hline Male & 35.4 (29.1 to 42.4$)$ & 1 & 1 \\
\hline Female & 32.8 (28.1 to 37.8$)$ & $1.0(0.7$ to 1.3$)$ & 1.1 (0.8 to 1.6) \\
\hline \multicolumn{4}{|l|}{ Age group } \\
\hline$\leq 9$ & 42.6 (34.6 to 53.7) & 1 & 1 \\
\hline 10 to 19 & 35.9 (28.0 to 44.7$)$ & 0.8 (0.5 to 1.3$)$ & $0.7(0.3$ to 1.1$)$ \\
\hline 20 to 29 & 23.9 (17.0 to 32.5$)$ & 0.5 (0.3 to 0.8$)$ & 0.4 (0.2 to 1.0$)$ \\
\hline 30 to 39 & 31.1 (23.9 to 39.4) & 0.7 (0.4 to 1.1$)$ & 0.8 (0.4 to 1.2$)$ \\
\hline 40 to 49 & 36.2 (26.4 to 47.3 ) & 0.8 (0.4 to 1.3$)$ & 0.8 (0.4 to 1.4$)$ \\
\hline $50 \leq$ & 25.4 (16.4 to 37.2$)$ & $0.6(0.3$ to 1.1$)$ & 0.5 (0.3 to 1.2$)$ \\
\hline \multicolumn{4}{|l|}{ Occupation } \\
\hline Housewife & 30.6 (24.1 to 38.0$)$ & 1 & 1 \\
\hline Children & 32.4 (24.5 to 41.4) & 1.0 (0.6 to 1.7$)$ & $2.2(0.5$ to 3.8$)$ \\
\hline Student & 45.8 (36.0 to 56.1 ) & 1.4 (0.9 to 2.1$)$ & 1.1 (0.5 to 2.1) \\
\hline Farmer & 32.3 (24.6 to 41.0$)$ & 1.1 (0.7 to 1.7$)$ & $1.0(0.5$ to 1.7$)$ \\
\hline Employed & 20.3 (12.0 to 32.1) & $0.6(0.3$ to 1.1$)$ & 0.6 (0.3 to 1.3$)$ \\
\hline Unemployed & $47.0(26.2$ to 68.8$)$ & 1.4 (0.6 to 3.1$)$ & 1.3 (0.5 to 3.5$)$ \\
\hline Others & 27.6 (17.7 to 40.5$)$ & $0.8(0.4$ to 1.4$)$ & 0.6 (0.3 to 1.2$)$ \\
\hline \multicolumn{4}{|l|}{ Education level } \\
\hline High school & 29.7 (22.2 to 38.4$)$ & 1 & 1 \\
\hline Children under 6-yr & 38.5 (29.3 to 48.6$)$ & 0.7 (0.4 to 1.1) & 0.1 (0.1 to 0.6$)$ \\
\hline Elementary school & 35.1 (29.0 to 41.7) & 0.9 (0.6 to 1.3$)$ & $0.7(0.5$ to 1.1$)$ \\
\hline University & 22.0 (12.3 to 36.1$)$ & $0.4(0.2$ to 0.9$)$ & 0.5 (0.2 to 1.2$)$ \\
\hline Illiterate & 33.0 (25.6 to 41.2$)$ & 0.8 (0.5 to 1.3$)$ & 0.7 (0.4 to 1.2$)$ \\
\hline \multicolumn{4}{|l|}{ Source of drinking water } \\
\hline Tap water & 31.6 (26.4 to 37.3$)$ & 1 & 1 \\
\hline Spring or well water & 38.4 (32.6 to 44.6$)$ & 1.3 (1.0 to 1.8$)$ & 1.3 (1.0 to 1.8$)$ \\
\hline \multicolumn{4}{|l|}{ Animal close contact } \\
\hline No & 32.7 (27.6 to 38.2$)$ & 1 & 1 \\
\hline Yes & $34.9(29.4$ to 40.9$)$ & 1.1 (0.8 to 1.4$)$ & $1.0(0.7$ to 1.3$)$ \\
\hline \multicolumn{4}{|l|}{ Residency status } \\
\hline Rural & 34.8 (30.5 to 39.3$)$ & 1 & 1 \\
\hline Urban & 28.6 (20.0 to 39.0$)$ & 0.6 (0.3 to 0.9$)$ & 0.4 (0.2 to 0.7$)$ \\
\hline
\end{tabular}

${ }^{\text {aCCrude odds ratio }}$

${ }^{b}$ Adjusted odds ratio

Indicates $p<0.05$

infrastructure, disorganization in health services and lower socioeconomic living conditions [25]. Several studies on human parasitic infections have revealed a common association between parasitic infections and lower socioeconomic status of rural area in Iran $[5,6,26]$.

In the current study, intestinal parasitic infections showed no significant association with sex, age group, occupation, education level, and animal close contact. In rural life of Rudbar-e Jonub, most women are involved in outdoor activities including farming and animal husbandry as like as men which exposes them to infection as much as men. Also, in this county, most housewives and students are involved in farming and animal husbandry and the occupational variation is low. Therefore, it is complex to discuss the association between sex and occupation with intestinal parasitic infections in this area. According to current finding, there was no significant association between sex and occupation with the infection, similar most studies carried out on the prevalence of intestinal parasites [9-11, 15]. 
The prevalence of parasitic infections in the age group $\leq 9$ year was $42.6 \%$ (95\% CI 34.6 to $53-7 \%$ ), higher than other groups but there was no statistically significant association between age groups and parasitic infections. The most common intestinal parasite in this age group was G. lamblia. Univariate analysis showed a significant association between Giardia infection and age group. It seems the lower levels of personal hygiene in children can be attributed to the higher prevalence of intestinal parasites in them. Regarding the participant's education level, although no significant association was found between the prevalence of intestinal parasitic infections and level of education, the results of this study indicate that as the level of literacy increases, the rate of parasitic infection decreases. Educated people are more aware of the transmission of parasitic infection and they may apply the necessary measurements to avoid the infection. Also, the results of this study showed the odds ratio of the infection in participants with and without close contact with the animal was almost same. These results indicate that domestic animals do not play a bold role in the transmission of intestinal parasites detected in this study to humans in Rudbar-e Jonub county.

\section{Limitations}

Because of cultural reasons, many households were reluctant to give their specimens which led to a low response rate $(57.4 \%)$ and also, stool specimens were collected once from each participant whereas for standard diagnosis of intestinal parasites, at least three specimens in three alternate days are necessary and also Graham test for E. vermicularis diagnosis was not done. Furthermore, because of financial constraints and limited facilities, molecular methods for identification of $E$. histolytica/dispar complex was not done. Another limitation of this study was the lack of collecting information on clinical symptoms.

\section{Conclusions}

This study revealed a high prevalence of intestinal protozoan infections among inhabitants of Rudbar-e Jonub county. Despite the downtrend of parasitic infections in Iran, compared to past decades, intestinal parasites especially protozoans remain a challenging public health problem wherever sanitation and health measures are limited.

\section{Abbreviations}

Cl: Confidence interval; MIF: Merthiolate-iodine-formaldehyde; OR: Odds ratio; STH: Soil-transmitted helminth

\section{Acknowledgments}

The authors thank Rudbar-e Jonub Health Center staff for their bits of help in collecting the samples.

\section{Authors' contributions}

MJAA, MB, M.R and MM designed the study. MJAA Writing original draft, VB carried out the statistical analysis, SA, MA, RH and MS collected the specimens and carried out the experiments. All authors read and approved the final version of the manuscript.

\section{Funding}

This study was funded by Jiroft University of Medical Sciences (Grant No. p96-53) to cover the fees for the design of the study, sample collection and laboratory analysis. But it had no role in analysis, interpretation of data and writing the manuscript. The author also declares that no other financial fund was secured from other sources for this research work.

\section{Availability of data and materials}

The datasets generated and/or analyzed during the current study may be made available from the corresponding authors on reasonable request.

\section{Ethics approval and consent to participate}

Informed written consent was obtained from the participants or their parents, in term of children. The study was approved by the Ethics Committee of the Jiroft University of Medical Sciences. Confidentiality of the details of the participants was assured.

\section{Consent for publication}

Not applicable.

\section{Competing interests}

The authors declare that they have no competing interests.

\section{Author details}

'Department of Medical Parasitology and Mycology, School of Public Health, Tehran University of Medical Sciences, Tehran, Iran. ${ }^{2}$ Health Affairs, Jiroft University of Medical Sciences, Jiroft, Iran. ${ }^{3}$ Sina Trauma and Surgery Research Center, Tehran University of Medical Sciences, Tehran, Iran.

Received: 26 September 2019 Accepted: 23 December 2019

Published online: 06 January 2020

\section{References}

1. WHO. World health organization (WHO), Amoebiasis. Weekly Epidemiol Rec. 1997;72:97-100 Sudan University of Science and Technology Questionnair.

2. Haque R. Human intestinal parasites. J Health Popul Nutr. 2007;25(4):387.

3. Nasiri V, Esmailnia K, Karim G, Nasir M, Akhavan O. Intestinal parasitic infections among inhabitants of Karaj City, Tehran province, Iran in 20062008. Korean J Parasitol. 2009;47(3):265.

4. Pestehchian N, Nazari M, Haghighi A, Salehi M, Yosefi HA, Khosravi N. Prevalence of intestinal parasitic infection among inhabitants and tribes of Chelgerd, Iran, 2008-2009. J Clin Diagn Res. 2015:9(5):LC01.

5. Haghighi A, Khorashad AS, Mojarad EN, Kazemi B, Nejad MR, Rasti S. Frequency of enteric protozoan parasites among patients with gastrointestinal complaints in medical centers of Zahedan, Iran. Trans R Soc Trop Med Hyg. 2009;103(5):452-4.

6. Kuzehkanani AB, Rezaei S, Babaei Z, Niyyati M, Hashemi S, Rezaeian M. Enteric protozoan parasites in rural areas of Bandar-Abbas, southern Iran: comparison of past and present situation. Iran J Public Health. 2011:40(1):80.

7. https://www.amar.org.ir/Portals/1/census/2016/Census_2016_Selected_ Findings.pdf. (Published: August 20, 2018).

8. Garcia LS, Arrowood M, Kokoskin E, Paltridge GP, Pillai DR, Procop GW, et al. Laboratory diagnosis of parasites from the gastrointestinal tract. Clin Microbiol Rev. 2018:31(1):e00025-17.

9. Mahni MB, Rezaeian M, Eshrat Beigom K, Raeisi A, Khanaliha K, Tarighi F, et al. Prevalence of intestinal parasitic infections in Jiroft, Kerman Province, Iran. Iran J Parasitol. 2016;11(2):232

10. Mowlavi G, MirAhmadi H, Rezaeian M, Kia E, Rokni M, Golestan B, et al. Prevalence of intestinal parasites in tribal parts of Khuzestan Province during 2005-07. Govaresh. 2008;12(4):219-28.

11. Kia E, Hosseini M, Nilforoushan M, Meamar A, Rezaeian M. Study of intestinal protozoan parasites in rural inhabitants of Mazandaran province, Northern Iran. Iran J Parasitol. 2008;3(1):21-5.

12. Sarkari B, Hosseini G, Motazedian MH, Fararouei M, Moshfe A. Prevalence and risk factors of intestinal protozoan infections: a population-based study 
in rural areas of Boyer-Ahmad district, southwestern Iran. BMC Infect Dis. 2016;16(1):703

13. Jafari R, Fallah M, Yousofi Darani H, Yousefi HA, Mohaghegh MA, Latifi M, et al. Prevalence of intestinal parasitic infections among rural inhabitants of Hamadan city, Iran, 2012. Avicenna J Clin Microbiol Infect. 2014;1(2):e21445.

14. Badparva E, Kheirandish F, Ebrahimzade F. Prevalence of intestinal parasites in Lorestan Province, west of Iran. Asian Pac J Trop Dis. 2014;4:S728-S32.

15. Hemmati N, Razmjou E, Hashemi-Hafshejani S, Motevalian A, Akhlaghi L, Meamar AR. Prevalence and risk factors of human intestinal parasites in Roudehen, Tehran province, Iran. Iran J Parasitol. 2017;12(3):364.

16. Nazer H, Greer W, Donnelly K, Mohamed A, Yaish H, Kagalwalla A, et al. The need for three stool specimens in routine laboratory examinations for intestinal parasites. Br J Clin Pract. 1993:47(2):76-8.

17. Bálint A, Dóczi I, Bereczki L, Gyulai R, Szúcs M, Farkas K, et al. Do not forget the stool examination! - cutaneous and gastrointestinal manifestations of Blastocystis sp. infection. Parasitol Res. 2014;113(4):1585-90.

18. Matiut DS, Hritcu L. The pathogenic role of Blastocystis isolated from patients with irritable bowel syndrome and colitis from lasi, Romania. Acta parasitologica. 2014;60(1):116-23.

19. Tan KS, Mirza H, Teo JD, Wu B, MacAry PA. Current views on the clinical relevance of Blastocystis spp. Curr Infect Dis Rep. 2010;12(1):28-35.

20. Engsbro AL, Stensvold CR, Nielsen HV, Bytzer P. Prevalence, incidence, and risk factors of intestinal parasites in Danish primary care patients with irritable bowel syndrome. Scand J Infect Dis. 2014:46(3):204-9.

21. Rokni M. The present status of human helminthic diseases in Iran. Ann Trop Med Parasitol. 2008;102(4):283-95.

22. Jalayer T, Farid H, Katiraei A. Prevalence of intestinal parasitic infection in Dorchepiaz, Isfahan. Iran J Public Health. 1987;6(1):9-15.

23. Rezaeian M, Saraei M. A survey of the prevalence of human parasites in rural areas of Lahijan. Iran J Public Health. 1992;4(1):29-35.

24. Rezaiian $\mathrm{M}$, Hooshyar $\mathrm{H}$. The prevalence of intestinal parasitic infection in rural areas of Tonekabon, Iran. Iran J Public Health. 1996;25(3):47-58

25. Balcioglu IC, Kurt Ö, Limoncu ME, Dinç G, Gümüș M, Kilimcioglu AA, et al. Rural life, lower socioeconomic status and parasitic infections. Parasitol Int. 2007:56(2):129-33

26. Nematian J, Nematian E, Gholamrezanezhad A, Asgari AA. Prevalence of intestinal parasitic infections and their relation with socio-economic factors and hygienic habits in Tehran primary school students. Acta Trop. 2004; 92(3):179-86.

\section{Publisher's Note}

Springer Nature remains neutral with regard to jurisdictional claims in published maps and institutional affiliations.

Ready to submit your research? Choose BMC and benefit from:

- fast, convenient online submission

- thorough peer review by experienced researchers in your field

- rapid publication on acceptance

- support for research data, including large and complex data types

- gold Open Access which fosters wider collaboration and increased citations

- maximum visibility for your research: over $100 \mathrm{M}$ website views per year

At $\mathrm{BMC}$, research is always in progress.

Learn more biomedcentral.com/submissions 